\title{
A Note on Knowledge-Based Programs and Specifications
}

\author{
Joseph Y. Halpern* \\ Computer Science Dept. \\ Cornell University \\ Ithaca, NY 14853 \\ halpern@cs.cornell.edu \\ http://www.cs.cornell.edu/home/halpern
}

\begin{abstract}
Knowledge-based program are programs with explicit tests for knowledge. They have been used successfully in a number of applications. Sanders has pointed out what seem to be a counterintuitive property of knowledge-based programs. Roughly speaking, they do not satisfy a certain monotonicity property, while standard programs (ones without tests for knowledge) do. It is shown that there are two ways of defining the monotonicity property, which agree for standard programs. Knowledge-based programs satisfy the first, but do not satisfy the second. It is further argued by example that the fact that they do not satisfy the second is actually a feature, not a problem. Moreover, once we allow the more general class of knowledge-based specifications, standard programs do not satisfy the monotonicity property either.
\end{abstract}

\footnotetext{
${ }^{*}$ Much of this work was carried out while the author was at the IBM Almaden Research Center. IBM's support is gratefully acknowledged. The work was also supported in part by NSF under grant IRI-96-25901, and by the Air Force Office of Scientific Research under contract F49620-91-C-0080 and grant F49620-96-1-0323.
} 


\section{Introduction}

Consider a simple program such as This program, denoted $\mathrm{Pg}_{1}$ for future

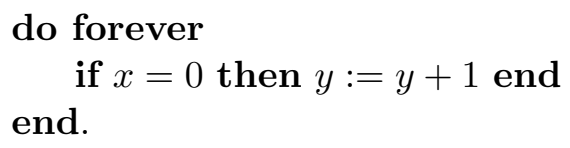

do forever

if $x=0$ then $y:=y+1$ end

end.

Figure 1: The program $\mathrm{Pg}_{1}$

reference, describes an action that a process (or agent-I use the two words interchangeably here) should take, namely, setting $y$ to $y+1$, under certain conditions, namely, if $x=0$. One way to way to provide formal semantics for such a program is to assume that each agent is in some local state, which, among other things, describes the value of the variables of interest. For this simple program, we need to assume that the local state contains enough information to determine the truth of the test $x=0$. We can then associate with the program a protocol, that is, a function describing what action the agent should in each local state. Note that a program is a syntactic object, given by some program text, while a protocol is a function, a semantic object.

Knowledge-based programs, introduced in [6, ,7] (based on the knowledgebased protocols of [9]) are intended to provide a high-level framework for the design and specification of protocols. The idea is that, in knowledge-based programs, there are explicit tests for knowledge. Thus, a knowledge-based program might have the form where $K(x=0)$ should be read as "you know $x=0$ ". We can informally view this knowledge-based program, denoted $\mathrm{Pg}_{2}$, as saying "if you know that $x=0$, then set $y$ to $y+1$ ". Roughly speaking, an agent knows $\varphi$ if, in all situations consistent with the agent's information, $\varphi$ is true.

Knowledge-based programs are an attempt to capture the intuition that what an agent does depends on what it knows. They have already met with

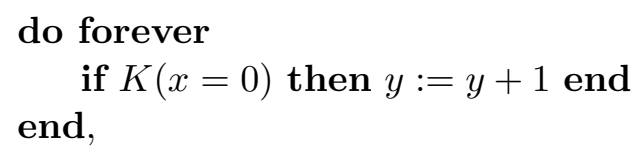

Figure 2: The program $\mathrm{Pg}_{2}$ 
some degree of success, having been used in papers such as [3, 8, 11, 12, 19, 17, 21, 22 both to help in the design of new protocols and to clarify the understanding of existing protocols. However, Sanders [25] has pointed out what seems to be a counterintuitive property of knowledge-based programs. Roughly speaking, she claims that knowledge-based programs do not satisfy a certain monotonicity property: a knowledge-based program can satisfy a specification under a given initial condition, but fail to satisfy it if we strengthen the initial condition. On the other hand, standard programs (ones without tests for knowledge) do satisfy the monotonicity property.

In this paper, I consider Sanders' claim more carefully. I show that it depends critically on what it means for a program to satisfy a specification. There are two possible definitions, which agree for standard programs. If we use the one closest in spirit to the ideas presented in [9], the claim is false, although it is true for the definition used by Sanders. But, even in the case of Sanders' definition, rather than being a defect of knowledge-base programs, this lack of monotonicity is actually a feature. In general, we do not want monotonicity. Moreover, once we allow a more general class of knowledge-based specifications, then standard programs do not satisfy the monotonicity property either.

The rest of this paper is organized as follows: In the next section, there is an informal review of the semantics of standard and knowledge-based programs. In Section [3, I discuss standard and knowledge-based specifications. In Section 1 , I consider the monotonicity property described by Sanders, and show in what sense it is and is not satisfied by knowledge-based programs. I give some examples in Section 5 showing why monotonicity is not always desirable. I conclude in Section 6 with some discussion of knowledge-based programs and specifications.

\section{Standard and knowledge-based programs: an informal review}

Formal semantics for standard and knowledge-based programs are provided in [6, 7]. To keep the discussion in this paper at an informal level, I simplify things somewhat here, and review what I hope will be just enough of the details so that, together with the examples given here, the reader will be able to follow the main points; the interested reader should refer to [6, [] for further discussion and all the formal details.

Informally, we view a distributed system as consisting of a number of 
interacting agents. We assume that, at any given point in time, each agent in the system is in some local state. A global state is just a tuple consisting of each agent's local state, together with the state of the environment, where the environment consists of everything that is relevant to the system that is not contained in the state of the processes. The agents' local states typically change over time, as a result of actions that they perform. A run is a function from time to global states. Intuitively, a run is a complete description of what happens over time in one possible execution of the system. A point is a pair $(r, m)$ consisting of a run $r$ and a time $m$. At a point $(r, m)$, the system is in some global state $r(m)$. For simplicity, time here is taken to range over the natural numbers (so that time is viewed as discrete, rather than continuous). A system $\mathcal{R}$ is a set of runs; intuitively, these runs describe all the possible executions of the system. For example, in a poker game, the runs could describe all the possible deals and bidding sequences.

Of major interest in this paper are the systems that we can associate with a program. To do this, we must first associate a system with a joint protocol. As was said in the introduction, a protocol is a function from local states to actions. (This function may be nondeterministic, so that in a given local state, there is a set of actions that may be performed.) A joint protocol is just a set of protocols, one for each process.

While the joint protocol describes what each process does, it does not give us enough information to generate a system. It does not tell us what the legal behaviors of the environment are, the effects of the actions, or the initial conditions. We specify these in the context. Formally, a context $\gamma$ is a tuple $\left(P_{e}, \mathcal{G}_{0}, \tau, \Psi\right)$, where $P_{e}$ is a protocol for the environment, $\mathcal{G}_{0}$ is a set of initial global states, $\tau$ is a transition function, and $\Psi$ is a set of admissible runs. The environment is viewed as running a protocol just like the agents; its protocol is used to capture features of the setting like "all messages are delivered within 5 rounds" or "messages may be lost". Given a joint protocol $P=$ $\left(P_{1}, \ldots, P_{n}\right)$ for the agents, an environment protocol $P_{e}$, and a global state $\left(s_{e}, s_{1}, \ldots, s_{n}\right)$, there is a set of possible joint actions $\left(\mathrm{a}_{e}, \mathrm{a}_{1}, \ldots, \mathrm{a}_{n}\right)$ that can be performed in this global state according to the protocols of the agents and the environment. (It is a set since the protocols may be nondeterministic.) The transition function $\tau$ describes how these joint actions change the global state by associating with each joint action a global state transformer, that is, a mapping from global states to global states. The set $\Psi$ of admissible runs is used to characterize notions like fairness. For the simple programs considered in this paper, the transition function will be almost immediate from the description of the global states and $\Psi$ will typically consist of all 
runs (so that it effectively plays no interesting role). What will change as we vary the context is the set of possible initial global states.

A run $r$ is consistent with joint protocol $P$ in context $\gamma$ if (1) $r(0)$, the initial global state of $r$, is one of the initial global states in $\mathcal{G}_{0},(2)$ for all $m$, the transition from global state $r(m)$ to $r(m+1)$ is the result of applying $\tau$ to a joint action that can be performed by $\left(P_{e}, P\right)$ in the global state $r(m)$, and (3) $r \in \Psi$. A system $\mathcal{R}$ represents a joint protocol $P$ in context $\gamma$ if it consists of all runs consistent with $P$ in $\gamma$.

Assuming that each test in a standard program run by process $i$ can be evaluated in each local state, we can derive a protocol from the program in an obvious way: to find out what process $i$ does in a local state $\ell$, we evaluate the tests in $\mathrm{Pg}$ in $\ell$ and perform the appropriate action. I $\mathrm{A}$ run is consistent with $\mathrm{Pg}$ in context $\gamma$ if it is consistent with the protocol derived from $\mathrm{Pg}$. Similarly, A system represents $\mathrm{Pg}$ in context $\gamma$ if it represents the protocol derived from $\mathrm{Pg}$. We use $\mathbf{R}(\mathrm{Pg}, \gamma)$ to denote the system representing $\mathrm{Pg}$ in context $\gamma$.

Example 2.1: Consider the simple standard program $\mathrm{Pg}_{1}$ in Figure 1 and suppose there is only one agent in the system. Further suppose the agent's local state is a pair of natural numbers $(a, b)$, where $a$ is the current value of variable $x$ and $b$ is the current value of $y$. The protocol derived from $\mathrm{Pg}_{1}$ increments the value of $b$ by 1 precisely if $a=0$. In this simple case, we can ignore the environment state, and just identify the global state of the system with the agent's local state. Suppose we consider the context $\gamma$ where the initial states consist of all possible local states of the form $(a, 0)$ for $a \geq 0$ and the transition function is such that the action $y:=y+1$ transforms $(a, b)$ to $(a, b+1)$. We ignore the environment protocol (or, equivalently, assume that $P_{e}$ performs the action no-op at each step) and assume $\Psi$ consist of all runs. A run $r$ is then consistent with $\operatorname{Pg}_{1}$ in context $\gamma$ if either (1) $r(0)$ is of the form $(0, b)$ and $r(m)$ is of the form $(0, b+m)$ for all $m \geq 1$, or $(2) r(m)$ is of the form $(a, b)$ for all $m$ and $a>0$. That is, either the $x$ component is originally 0 , in which case the $y$ component is continually increased by 1 , or else nothing happens.

Now we turn to knowledge-based programs. Here the situation is somewhat more complicated. In a given context, a process can determine the

\footnotetext{
${ }^{1}$ Strictly speaking, to evaluate the tests, we need an interpretation that assigns truth values to formulas in each global state. For the programs considered here, the appropriate interpretation will be immediate from the description of the system, so I ignore interpretations here for ease of exposition.
} 
truth of a test such as " $x=0$ " by simply looking at its local state. However, in a knowledge-based program, there are tests for knowledge. According to the definition of knowledge in systems, an agent $i$ knows a fact $\varphi$ at a given point $(r, m)$ in system $\mathcal{R}$ if $\varphi$ is true at all points in $\mathcal{R}$ in which $i$ has the same local state as it does at $(r, m)$. Thus, $i$ knows $\varphi$ at the point $(r, m)$ if $\varphi$ holds at all points consistent with $i$ 's information at $(r, m)$. The truth of a test for knowledge cannot in general be determined simply by looking at the local state in isolation. We need to look at the whole system. As a consequence, given a run, we cannot in general determine if it is consistent with a knowledge-based program in a given context. This is because we cannot tell how the tests for knowledge turn out without being given the other possible runs of the system; what a process knows at one point will depend in general on what other points are possible. This stands in sharp contrast to the situation for standard programs.

This means it no longer makes sense to talk about a run being consistent with a knowledge-based program in a given context. However, notice that, given a system $\mathcal{R}$, we can derive a protocol from a knowledge-based program $\mathrm{Pg}_{k b}$ for process $i$ by using $\mathcal{R}$ to evaluate the knowledge tests in $\mathrm{Pg}_{k b}$. That is, a test such as $K \varphi$ holds in a local state $l$ if $\varphi$ holds at all points in $\mathcal{R}$ where process $i$ has local state $l$. In general, different protocols can be derived from a given knowledge-based program, depending on what system we use to evaluate the tests. Let $\operatorname{Pg}_{k b}^{\mathcal{R}}$ denote the protocol derived from $\operatorname{Pg}_{k b}$ given system $\mathcal{R}$.

We say that a system $\mathcal{R}$ represents a knowledge-based program $\operatorname{Pg}_{k b}$ in context $\gamma$ if $\mathcal{R}$ represents the protocol $\operatorname{Pg}_{k b}^{\mathcal{R}}$. That is, $\mathcal{R}$ represents $\operatorname{Pg}_{k b}$ if $\mathcal{R}=\mathbf{R}\left(\operatorname{Pg}_{k b}^{\mathcal{R}}, \gamma\right)$. Thus, a system represents $\operatorname{Pg}_{k b}$ if it satisfies a certain fixed-point equation.

This definition is somewhat subtle, and determining the system representing a given knowledge-based program may be nontrivial. Indeed, as shown in [6, [], in general, there may be no systems representing a knowledge-based program $\mathrm{Pg}_{k b}$ in a given context, only one, or more than one, since the fixed-point equation may have no solutions, one solution, or many solutions. Moreover, computing the solutions may be a difficult task, even if we have only finitely many possible global states. There are conditions sufficient to guarantee that there is exactly one system representing $\mathrm{Pg}_{k b}$, and these conditions are satisfied by many knowledge-based programs of interest, and, in particular, by the programs discussed in this paper. If $\mathrm{Pg}_{k b}$ has a unique system representing it in context $\gamma$, then we again denote this system $\mathbf{R}\left(\operatorname{Pg}_{k b}, \gamma\right)$. 
Example 2.2: The knowledge-based program $\mathrm{Pg}_{2}$ in Figure 2, with the test $K(x=0)$, is particularly simple to analyze. If we consider the context $\gamma$ discussed in Example 2.1, then whether or not $x=0$ holds is determined by the process' local state. Thus, in context $\gamma, x=0$ holds iff $K(x=0)$ holds, and the knowledge-based program reduces to the standard program.

On the other hand, consider the context $\gamma^{\prime}$ where the agent's local state just consists just of the value of $y$, while the value of $x$ is part of the environment state. Again, we can identify the global state with a pair $(a, b)$, where $a$ is the current value of $x$ and $b$ is the current value of $y$, but now $a$ represents the environment's state, while $b$ represents the agent's state. We can again assume the environment performs the no-op action at each step, $\Psi$ consists of all runs, the transition function is as in Example 2.1, and the initial states are all possible global states of the form $(a, 0)$. In this context, there is a also unique system representing $\mathrm{Pg}_{2}$ : The agent never knows whether $x=0$, so there is a unique run corresponding to each initial state $(a, 0)$, in which the global state is $(a, 0)$ throughout the run.

Finally, let $\gamma^{\prime \prime}$ be identical to $\gamma^{\prime}$ except that the only initial state is $(0,0)$. Again, there will be a unique system representing $\mathrm{Pg}_{2}$ in $\gamma^{\prime \prime}$, but it is quite different from $\mathbf{R}\left(\mathrm{Pg}_{2}, \gamma^{\prime}\right)$. In $\mathbf{R}\left(\mathrm{Pg}_{2}, \gamma^{\prime \prime}\right)$, the agent knows that $x=0$ at all times. There is only one run, where the value of $y$ is augmented at every step.

This discussion suggests that a knowledge-based program can be viewed as specifying a set of systems, the ones that satisfy a certain fixed-point property, while a standard program can be viewed as specifying a set of runs, the ones consistent with the program.

\section{$3 \quad$ Standard and knowledge-based specifications}

Typically, we think of a protocol being designed to satisfy a specification, or set of properties. Although a specification is often written in some specification language (such as temporal logic), many specifications can usefully be viewed as predicates on runs. This means that we can associate a set of runs with a specification; namely, all the runs that satisfy the required properties. Thus, a specification such as "all processes eventually decide on the same value" would be associated with the set of runs in which the processes do all decide the same value.2

\footnotetext{
${ }^{2}$ Of course, there are useful specifications that cannot be viewed as predicates on runs. While linear time temporal logic assertions are predicates on runs, branching time tempo-
} 
Researchers have often focused attention on two types of specifications: safety properties - these are invariant properties that have the form "a particular bad thing never happens" - and liveness properties - these are properties that essentially say "a particular good thing eventually does happen" [24]. Thus, a run $r$ has a safety property $p$ if $p$ holds at all points $(r, m)$, while $r$ has the liveness property $q$ if $q$ holds at some point $(r, m)$. Suppose we are interested in a program that guarantees that all the processes eventually decide on the same value. We model this by assuming that each process $i$ has a decision variable $x_{i}$, initially undefined, in its local state (we can assume a special "undefined" value in the domain), which is set once in the course of a run, when the decision is made. Given the way we have chosen to model this problem, we would expect this program to satisfy two safety properties: (1) each process' decision variable is changed at most once (so that it is never the case that it is set more than once); and (2) if neither $x_{i}$ nor $x_{j}$ has value "undefined", then they are equal. We also expect it to satisfy one liveness property: each decision variable is eventually set.

We say that a standard program $\mathrm{Pg}$ satisfies a specification $\sigma$ in a context $\gamma$ if every run consistent with $\mathrm{Pg}$ in $\gamma$ (that is, every run in the system representing $\mathrm{Pg}$ in $\gamma$ ) satisfies $\sigma$. Similarly, we can say that a knowledgebased program $\operatorname{Pg}_{k b}$ satisfies specification $\sigma$ in context $\gamma$ if every run in every system representing $\mathrm{Pg}_{k b}$ satisfies $\sigma$.

The notion of specification we have considered so far can be thought of as being run based. A specification $\sigma$ is a predicate on (i.e., set of) runs and a program satisfies $\sigma$ if each run consistent with the program is in $\sigma$. Although run-based specifications arise often in practice, there are reasonable specifications that are not run based. There are times that it is best to think of a specification as being, not a predicate on runs, but a predicate on entire systems. For example, consider a knowledge base (KB) that responds to queries by users. We can imagine a specification that says "To a query

ral logic assertions are best viewed as predicates on trees. (See [4, 16] for a discussion of the differences between linear time and branching time.) For example, Koo and Toueg's notion of weak termination [14] requires that at every point there is a possible future where everyone terminates. In the notation used in this paper, this would mean that for every point $(r, m)$, there must be another point $\left(r^{\prime}, m\right)$ such that $r$ and $r^{\prime}$ are identical up to time $m$, and at some point $\left(r^{\prime}, m^{\prime}\right)$ with $m^{\prime} \geq m$, every process terminates. This assertion is easily expressed in branching time logic. Probabilistic assertions such as "all processes terminate with probability .99" also cannot be viewed as predicates on individual runs. Other examples of specifications that cannot be viewed as a predicate on runs are discussed later in this section. Nevertheless, specifications that are predicates on runs are sufficiently prevalent that it seems reasonable to give them special attention. 
of $\varphi$, answer 'Yes' if you know $\varphi$, answer 'No' if you know $\neg \varphi$, otherwise answer 'I don't know'." This specification is given in terms of the KB's knowledge, which depends on the whole system and cannot be determined by considering individual runs in isolation. We call such a specification a knowledge-based specification. Typically, we think of a knowledge-based specification being given as a formula involving operators for knowledge and time. Formally, it is simply a predicate on (set of) systems. (Intuitively, it consists of all the systems where the formula is valid-i.e., true at every point in the system.) $)^{3}$

We can think of a run-based specification $\sigma$ as a special case of a knowledgebased specification. It consists of all those systems all of whose runs satisfy $\sigma$. A (standard or knowledge-based) program Pg satisfies a knowledge-based specification $\sigma$ in context $\gamma$ if every system representing $\operatorname{Pg}$ in $\gamma$ satisfies the specification.

Notice that knowledge-based specifications bear the same relationship to (standard) specifications as knowledge-based programs bear to standard programs. A knowledge-based specification/program in general defines a set of systems; a standard specification/program defines a set of runs (i.e., a single system).

\section{Monotonicity}

Sanders 25] focuses on a particular monotonicity property of specifications. To understand this property, and Sanders' concerns, we first need some definitions. Given contexts $\gamma=\left(P_{e}, \mathcal{G}_{0}, \tau, \Psi\right)$ and $\gamma^{\prime}=\left(P_{e}^{\prime}, \mathcal{G}_{0}^{\prime}, \tau^{\prime}, \Psi^{\prime}\right)$, we write $\gamma^{\prime} \sqsubseteq \gamma$ if $P_{e}=P_{e}^{\prime}, \mathcal{G}_{0}^{\prime} \subseteq \mathcal{G}_{0}, \tau=\tau^{\prime}$, and $\Psi^{\prime} \subseteq \Psi$. That is, in $\gamma^{\prime}$ there may be fewer initial states and fewer admissible runs, but otherwise $\gamma$ and $\gamma^{\prime}$ are the same. The following lemma is almost immediate from the definitions.

Lemma 4.1: If $\gamma^{\prime} \sqsubseteq \gamma$, then for all protocols $P$, every run consistent with $P$ in $\gamma^{\prime}$ is also consistent with $P$ in $\gamma$, so $\mathbf{R}\left(P, \gamma^{\prime}\right) \subseteq \mathbf{R}(P, \gamma)$. Similarly, for every standard program $\mathrm{Pg}$, we have $\mathbf{R}\left(\mathrm{Pg}, \gamma^{\prime}\right) \subseteq \mathbf{R}(\mathrm{Pg}, \gamma)$.

The restriction in Lemma 4.1 to standard programs is necessary. It is not true for knowledge-based programs. The set of systems consistent with

\footnotetext{
${ }^{3}$ As the examples discussed in Footnote 2 show, not all predicates on systems can be expressed in terms of formulas involving knowledge and time. I will not attempt to characterize here the ones that can be so expressed. It is not even clear that such a characterization is either feasible or useful.
} 
a knowledge-based program can be rather arbitrary, as Example 2.2 shows. This example also shows that safety and liveness properties need not be preserved when we restrict the context. The safety property " $y$ is never equal to 1 " is satisfied by $\mathrm{Pg}_{2}$ in context $\gamma^{\prime}$ but not in context $\gamma^{\prime \prime}$. On the other hand, the liveness property " $y$ is eventually equal to 1 " is satisfied by $\mathrm{Pg}_{2}$ in context $\gamma^{\prime \prime}$ but not $\gamma^{\prime}$.

Sanders suggests that this behavior is somewhat counterintuitive. To quote [25]:

[A] knowledge-based protocol need not be monotonic with respect to the initial conditions ... [In particular,] safety and liveness properties of knowledge-based protocols need not be preserved by strengthening the initial conditions, thus violating one of the most intuitive and fundamental properties of standard programs [italics Sanders'].'t]

It is certainly true that the system representing a knowledge-based program in a restricted context is not necessarily a subset of the system representing it in the original context. However, under what is arguably the most natural interpretation of what it means for a program to satisfy a specification with respect to an initial condition, a knowledge-based program is monotonic with respect to initial conditions.

To understand why this should be so, we need to make precise what it means for a (knowledge-based) program to satisfy a specification with respect to an initial condition. Formally, we can take an initial condition to be a predicate on global states (so that an initial condition corresponds to a set of global states). An initial condition $I N I T^{\prime}$ is a strengthening of INIT if $I N I T^{\prime}$ is a subset of INIT. (In logical terms, this means that INIT' can be thought of as implying INIT.) A set $G$ of global states satisfies an initial condition INIT if $G \subseteq I N I T$.

Suppose that we fix $P_{e}, \tau$, and $\Psi$, that is, all the components of a context except the set of initial global states, and consider the family $\Gamma=$ $\Gamma\left(P_{e}, \tau, \Psi\right)$ of contexts of the form $\left(P_{e}, \mathcal{G}_{0}, \tau, \Psi\right)$, where the set $\mathcal{G}_{0}$ varies over all subsets of global states. Now it seems reasonable to say that program $\mathrm{Pg}$ satisfies specification $\sigma$ (with respect to $\Gamma$ ) given initial condition INIT if $\mathrm{Pg}$ satisfies $\sigma$ in every context in $\Gamma$ whose initial global states satisfy INIT.

${ }^{4}$ In [9], a notion of knowledge-based protocol was introduced, and Sanders is referring to that notion, rather than the notion of knowledge-based program that I am using here. See [7] for a discussion of the difference between the two notions. Sanders' comments apply without change to knowledge-based programs as defined here. 
With this definition, it is clear that if $\mathrm{Pg}$ satisfies $\sigma$ given INIT, and INIT is a strengthening of INIT, then $\mathrm{Pg}$ must also satisfy $\sigma$ with respect to $I N I T^{\prime}$, since every context whose initial global states are in $I N I T^{\prime}$ also has its initial global states in INIT.

Thus, under this definition of what it means for a program to satisfy a specification, Sanders' observation is incorrect. However, Sanders used a somewhat different definition. Suppose that rather than considering all contexts in $\Gamma$ whose initial global states satisfy INIT, we consider the maximal one, that is, the one whose set of initial global states consists of all global states in $\Sigma$ that satisfy INIT. We say that $\mathrm{Pg}$ maximally satisfies specification $\sigma$ (with respect to $\Gamma$ ) given INIT if $\mathrm{Pg}$ satisfies $\sigma$ in the context in $\Gamma$ whose set of initial global states consists of all global states satisfying INIT.

It is almost immediate from Lemma 4.1 and the definitions that for standard programs and standard specifications, "satisfaction with respect to $\Gamma$ " coincides with "maximal satisfaction with respect to $\Gamma$ ". On the other hand, they can be quite different for knowledge-based programs and knowledge-based specifications, as the following examples show.

Example 4.2: For the knowledge-based program $\mathrm{Pg}_{2}$, if we take $\Gamma$ to consist of all contexts $\left(P_{e}, \mathcal{G}_{0}, \tau, \Psi\right)$, where $P_{e}, \tau$, and $\Psi$ are as discussed in Example 2.2 and $\mathcal{G}_{0}$ is some subset of the global states, then, as we observed above, $\mathrm{Pg}_{2}$ satisfies the specification " $y$ is never equal to 1 " for the initial condition $I N I T_{1}$ which can be characterized by the formula $y=0$ but not for the initial condition $I N I T_{2}$ characterized by $x=0 \wedge y=0$. Similarly, if $\mathrm{Pg}_{3}$ is the result of replacing the test $K(x=0)$ in $\mathrm{Pg}_{2}$ by $\neg K(x=0)$, then $\mathrm{Pg}_{3}$ satisfies the liveness condition " $y$ is eventually equal to 1 " for $I N I T_{1}$ but not for $I N I T_{2}$. This shows that a standard specification (in particular, one involving safety or liveness) may not be monotonic with respect to maximal specification for a knowledge-based program.

Example 4.3: Consider the standard program $\mathrm{Pg}_{1}$ again, but now consider a context where there are two agents. Intuitively, the second agent never learns anything and plays no role. Formally, this is captured by taking the second agent's local state to always be $\lambda$. Thus, a global state now has the form $(\langle a, b\rangle, \lambda)$. We can again identify the global state with the local state of the first agent (the one performing all the actions). Thus, abusing notation somewhat, we can consider the same set of contexts as in Example 4.2. Now consider the knowledge-based specification $K_{2}(y=0)$. This is true with 
respect to $\Gamma$ for the initial condition $I N I T_{1}$ but not for $I N I T_{2}$. This shows that even for a standard program, a knowledge-based specification may not be monotonic with respect to maximal satisfaction.

Example 4.4: In the muddy children problem discussed in [10], the father of the children says "Some [i.e., one or more] of you have mud on your forehead." The father then repeatedly asks the children "Do you know that you have mud on your own forehead?" Thus, the children can be viewed as running a knowledge-based program according to which a child answers "Yes" iff she knows that she has mud on her forehead. The father's initial statement is taken to restrict the possible initial global states to those where one or more children have mud on their foreheads. It is well known that, under this initial condition, the knowledge-based program satisfies the liveness property "all the children with mud on their foreheads eventually know it". On the other hand, if the father instead gives the children more initial information, by saying "Child 1 has mud on his forehead" (thus restricting the set of initial global states to those where child 1 has mud on his forehead), none of the children that have mud on their forehead besides child 1 will be able to figure out that they have mud on their forehead. Roughly speaking, this is because the information available to the children from child 1 's "No" answer in the original version of the story is no longer available once the father gives the extra information. (See [6, Example 7.25].) This problem is not an artifact of using knowledge-based programs or specifications. Rather, it is really the case in the original puzzle that if the father had said "Child 1 has mud on his forehead" rather than "Some of you have mud on your foreheads", the children with mud on their foreheads would never be able to figure out that they had mud on their foreheads. Sometimes extra knowledge can be harmful!

As should be clear from the preceding discussion, there are two notions of monotonicity, which happen to coincide (and hold) for standard programs and specifications, but differ if we consider knowledge-based programs or knowledge-based specifications. For knowledge-based programs and specifications, the first notion of monotonicity holds, while the second (monotonicity with respect to maximal satisfaction) does not. Monotonicity is

\footnotetext{
${ }^{5}$ Another example of the phenomenon that extra knowledge can be harmful can be found in 20. This is also a well-known phenomenon in the economics/game theory literature 23].
} 
certainly a desirable property - for a monotonic specification and program, once we prove that the specification holds for the program for a given initial condition, then we can immediately conclude that it holds for all stronger specifications. Without monotonicity, one may have to reprove the property for all stronger initial conditions. Maximal satisfaction also certainly seems like a reasonable generalization from the standard case. Thus, we should consider to what extent it is a problem that we lose monotonicity for maximal satisfaction when we consider knowledge-based programs and specifications.

Of course, whether something is problematic is, in great measure, in the eye of the beholder. Nevertheless, I would claim that, in the case of maximal satisfaction, the only properties that are lost when the initial condition is strengthened are either unimportant properties, or properties that, roughly speaking, ought to be lost. More precisely, they are properties that happen to be true of a particular context, but are not intrinsic properties of the program. The examples and the technical discussion below should help to make the point clearer. Thus, this lack of monotonicity should not be viewed as a defect of knowledge-based programs and specifications. Rather, it correctly captures the subtleties of knowledge acquisition in certain circumstances.

\section{Some examples}

Consider again the program $\mathrm{Pg}_{2}$. It can be viewed as saying "perform a sequence of actions (continually increasing $y$ ) if you know that $x=0$ ". In the system $\mathbf{R}\left(\mathrm{Pg}_{2}, \gamma^{\prime}\right)$, the initial condition guarantees that the agent does not know the value of $x$, and thus nothing is done. The strengthening of the initial condition to $x=0 \wedge y=0$ described by $\gamma^{\prime \prime}$ guarantees that the agent does know that $x=0$, and thus actions are performed. In this case, we surely do not want a safety condition like " $y$ is never equal to 1 ", which holds if the sequence of actions is not performed, to be preserved when we strengthen the initial condition in this way. Similarly, for the program $\mathrm{Pg}_{3}$ defined in Example 4.2, where the action is performed if the agent does

not know that $x=0$, we would not expect a liveness property like " $y$ is eventually equal to 1" to be preserved.

Clearly, there are times when we would like a safety or a liveness property to be preserved when we strengthen initial conditions. But these safety or liveness properties are typically ones that we want to hold of all systems consistent with the knowledge-based program, not just the ones represent- 
ing the program in certain maximal contexts. The tests in a well-designed knowledge-based program are often there precisely to ensure that desired safety properties do hold in all systems consistent with the program. For example, there may be a test for knowledge to ensure that an action is performed only if it is known to be safe (i.e., it does not violate the safety property). It is often possible to prove that such safety properties hold in all systems consistent with the knowledge-based program; thus, the issue of needing to reprove the property if we strengthen the initial conditions does not arise. (See [6, pp. 259-270] for further discussion of this issue.)

In the case of liveness properties, we often want to ensure that a given action is eventually performed. It is typically the case that an action in a knowledge-based program is performed when a given fact is known to be true. Thus, the problem reduces to ensuring that the knowledge is eventually obtained. As a consequence, the knowledge-based approach often makes it clearer what is required for the liveness property to hold. One example of how safety properties can be ensured by appropriate tests for knowledge and how liveness properties reduce to showing that a certain piece of knowledge is eventually obtained is given by the knowledge-based programs of [12]. I illustrate these points here using a simpler example.

Suppose we have a network of $n$ processes, connected via a communication network. The network is connected, but not necessarily completely connected. For simplicity, assume each communication link is bidirectional. We assume that all messages arrive within one time unit. Each process knows which processes it is connected to; formally, this means that the local state of each process includes a mapping associating each outgoing link with the identity of the neighbor at the other end. We also assume that each process records in its local state the messages it has sent and received. We want a program for process 1 to broadcast a binary value to all the processes in the network. Formally, we assume that each process $i$ has a local variable, say $x_{i}$, which is intended to store the value. The specification that the program must satisfy consists of three properties. For every run, and for all $i=1, \ldots, n$, we require the following:

1. $x_{i}$ changes value at most once,

2. $x_{1}$ never changes value, and

3. eventually the value of $x_{i}$ is equal to that of $x_{1}$.

Note that the first two properties are safety properties, and the last is a liveness property. 
A simple standard program that satisfies this specification is for process 1 to send $v$, the value of $x_{1}$, to all its neighbors; then the first time process $i(i \neq 1)$ gets the value $v$, it sets $x_{i}$ to $v$ and sends $v$ to all its neighbors except the one from which it received the message. Process $i$ does nothing if it later gets the value $v$ again. This program is easily seen to satisfy the specification in the context implicitly described above. We remark that, in principle, we could modify the first property to allow $x_{1}$ to change value a number of times before finally "stabilizing" on a final value. However, allowing this would only complicate the description of the property, since we would have to modify the third property to guarantee that the value of $x_{i}$ after stabilizing is equal to that of $x_{1}$. We return to this point below.

The behavior of each process can easily be captured in terms of knowledge: When a process knows the value of $x_{1}$, it sends the value to all its neighbors except those that it knows already know the value of $x_{1}$. Let $K_{i}\left(x_{1}\right)$ be an abbreviation for "process $i$ knows the value of $x_{1}$ ". (Thus, $K_{i}\left(x_{1}\right)$ is an abbreviation for $K_{i}\left(x_{1}=0\right) \vee K_{i}\left(x_{1}=1\right)$.) Similarly, let $K_{i} K_{j}\left(x_{1}\right)$ be an abbreviation for "process $i$ knows that process $j$ knows the value of $x_{1}$." Then we have the joint knowledge-based program DIFFUSE =

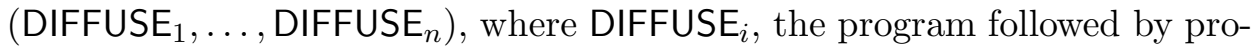
cess $i$, is

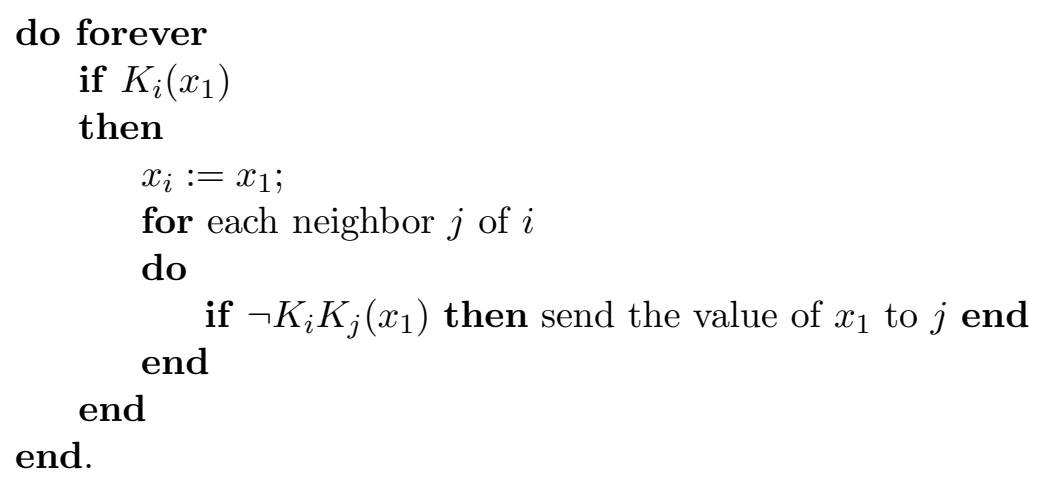

By considering this knowledge-based program, we abstract away from the details of how $i$ gains knowledge of the value of $x_{1}$. If $i=1$, then presumably the value was known all along; otherwise it was perhaps acquired through the receipt of a message. Similarly, the fact that $i$ sends the value of $x_{1}$ to a neighbor $j$ only if $i$ doesn't know that $j$ knows the value of $x_{1}$ handles two of the details of the standard program: (1) it guarantees that $i$ does not send the value of $x_{1}$ to $j$ if $i$ received the value of $x_{1}$ from $j$, and (2) it guarantees that $i$ does not send the value of $x_{1}$ to its neighbors more than 
once. F Finally, observe that DIFFUSE is correct even if messages can be lost, as long as the system satisfies an appropriate fairness assumption (if a message is sent infinitely often, it will eventually be delivered). 1 In this case process $i$ would keep sending the value of $x_{1}$ to $j$ until $i$ knows (perhaps by receiving an acknowledgment from $j$ ) that $j$ knows the value of $x_{1}$. The fact that DIFFUSE is correct "even if messages can be lost" or "no matter what the network topology" means that the program meets its specification in a number of different contexts.

This knowledge-based program has another advantage: it suggests ways to design more efficient standard programs. For example, process $i$ does not have to send the value of $x_{1}$ to all its neighbors (except the one from which it received the value of $x_{1}$ ) if it has some other way of knowing that a neighbor already knows the value of $x_{1}$. This may happen if the value of $x_{1}$ has a header describing to which processes it has already been sent. It might also happen if the receiving process has some knowledge of the network topology (for example, there is no need to rebroadcast the value of $x_{1}$ if communication is reliable and all processes are neighbors of process 1 ).

Returning to our main theme, notice that in every context $\gamma$ consistent with our assumptions, in the system(s) representing DIFFUSE in $\gamma$, the three properties described above are satisfied: $x_{i}$ changes value at most once in any run, $x_{1}$ never changes value, and eventually the value of $x_{i}$ is equal to that of $x_{1}$. Notice also the role of the test $K_{i}\left(x_{1}\right)$ in ensuring that the safety properties hold. As a result of the test, we know that $x_{i}$ is not updated until the value of $x_{1}$ is known; when it is updated, it is set to $x_{1}$. This guarantees that $x_{1}$ never changes value, and that $x_{i}$ changes value at most once and, when it does, it is set to $x_{1}$. All that remains is to guarantee that $x_{i}$ is eventually set to $x_{1}$. What the knowledge-based program makes clear is that this amounts to ensuring that all processes eventually know the value of $x_{1}$. It is easy to prove that this is indeed the case.

It is also easy to see that there are other properties that do not hold in all contexts. For a simple example, suppose that $n=3$, so there are

\footnotetext{
${ }^{6}$ This argument depends in part on our assumption that process $i$ is keeping track of the messages it sends and receives. If $i$ forgets the fact that it received the value of $x_{1}$ from $j$ then (if $i$ follows DIFFUSE ${ }_{i}$ ), it would send the value of $x_{1}$ back to $j$. Similarly, if $i$ receives the value of $x_{1}$ a second time and forgets that it has already sent it once to its neighbors, then according to DIFFUSE ${ }_{i}$, it would send it again. In addition, the assumption that there are no process failures is crucial.

${ }^{7}$ Note that this fairness assumption can be captured by using an appropriate set $\Psi$ (consisting only of runs where the fairness condition is satisfied) in the context.
} 
three processes in the network. Suppose that there is a link from process 1 to process 2 , and a link from process 2 to process 3 , and that these are the only links in the network. Moreover, suppose that the network topology is common knowledge. Given these simplifying assumptions, a process $i$ 's initial state consists of an encoding of the network topology, its name, and the value of $x_{i}$. Now consider two contexts: in context $\gamma_{1}$, there are 8 initial global states, in which $\left(x_{1}, x_{2}, x_{3}\right)$ take on all values in $\{0,1\}^{3}$; in $\gamma_{2}$, there are 4 initial global states, in which $\left(x_{1}, x_{2}, x_{3}\right)$ take on all values in $\{0,1\}^{3}$ such that $x_{1}=x_{3}$. Intuitively, in context $\gamma_{2}$, process 3 knows the value of $x_{1}$ (since it is the same as the value of $x_{3}$, which is part of process 3 's initial state), while in $\gamma_{1}$, neither process 2 nor process 3 know the value of $x_{1}$. Let $\mathcal{R}_{1}=\mathbf{R}\left(\right.$ DIFFUSE, $\left.\gamma_{1}\right)$ and let $\mathcal{R}_{2}=\mathbf{R}\left(\right.$ DIFFUSE, $\left.\gamma_{2}\right)$. It is not hard to see that $\mathcal{R}_{1}$ has eight runs, one corresponding to each initial global state. In each of these runs, process 1 sends the value of $x_{1}$ to process 2 in round 1; process 2 sets $x_{2}$ to this value in round 2 and forwards the value to process 3 ; in round 3 , process 3 sets $x_{3}$ to $i$ (and sends no messages). (Note that, formally, round $k$ takes place between times $k-1$ and $k$.) Similarly, $\mathcal{R}_{2}$, has four runs, one corresponding to each initial global state. In these runs, process 3 initially knows the value of $x_{1}$, although process 2 does not. Moreover, process 2 knows this. Thus, in the round of the runs in $\mathcal{R}_{2}$, both process 1 and process 3 send the value of $x_{1}$ to process 2 . But now, process 2 does not send a message to process 3 in the second round.

As expected, we can observe that not all liveness properties are preserved as we move from $\mathcal{R}_{1}$ to $\mathcal{R}_{2}$. For example, the runs in $\mathcal{R}_{1}$ all satisfy the liveness property "eventually process 2 sends a message to process 3 ". Clearly the runs in $\mathcal{R}_{2}$ do not satisfy this liveness property. This should be seen as a feature, not a bug! There is no reason to preserve the sending of unnecessary messages. The extra knowledge obtained when the initial conditions are strengthened may render sending the message unnecessary.

\section{Discussion}

When designing programs, we often start with a specification and try to find an (easily-implementable) standard program that satisfies it. The process of going from a specification to an implementation is often a difficult one. I would argue that quite often it is useful to express the properties we desire using a knowledge-based specification, proceed from there to construct a

knowledge-based program, and then go from the knowledge-based program 
to a standard program. While this approach may not always be helpful (indeed, if a badly designed knowledge-based program is used, then it may actually be harmful), there is some evidence showing that it can help.

The first examples of going from knowledge-based specifications to (standard) programs can be found in 11, 3, 15] (although the formal model used in [1, 15] is somewhat different from that described here). The approach described here was used in [12] to derive solutions to the sequence transmission problem (the problem of transmitting a sequence of bits reliably over a possibly faulty communication channel). All the programs derived in [12] are (variants of) well-known programs that solved the problem. While I would argue that the knowledge-based approach shows the commonality in the approaches used to solve the problem, and allows for easier and more uniform proofs of correctness, certainly this example by itself is not convincing evidence of the power of the knowledge-based approach.

Perhaps more convincing evidence is provided by the results of [3, 11, 21], where this approach is used to derive programs that are optimal (in terms of number of rounds required) for Byzantine Agreement and Eventual Byzantine Agreement. In this case, the programs derived were new, and it seems that it would have been quite difficult to derive them directly from the original specifications.

Knowledge-based specifications are more prevalent than it might at first seem. We are often interested in constructing programs that not only satisfy some safety and liveness conditions, but also use a minimal number of messages or rounds. As we have already observed, specifications of the form "do not send unnecessary messages" are not standard specifications; the same is true for a specification of the form "halt as soon as possible". Such specifications can be viewed as knowledge-based specifications. The results of [3, 11, 21] can be viewed as showing how knowledge-based specifications arise in the construction of round-efficient programs. The tests for knowledge in the knowledge-based programs described in these papers explicitly embody the intuition that a process decides as soon as it is safe to do so.

Similar sentiments about the importance of knowledge-based specifications are expressed by Mazer [18] (although the analogy between knowledgebased programs and knowledge-based specifications is not made in that paper):

Epistemic [i.e., knowledge-based] specifications are surprisingly common: a problem specification that asserts that a property or value is private to some process is an epistemic specifica- 
tion (e.g., "each database site knows whether it has committed the transaction"). We are also interested in epistemic properties to capture assertions on the extent to which a process's local state accurately reflects aspects of the system state, such as "each database site knows whether the others have committed the transaction".

For another example of the usefulness of knowledge-based specifications, recall our earlier discussion of the specification of the program for broadcasting a message through a network. If we replace the liveness requirements by the simple knowledge-based requirement "eventually process $i$ knows the value of $x_{1}$ ", we can drop the first property (that $x_{i}$ changes value at most once) altogether. Indeed, we do not have to mention $x_{i}, i \neq 1$, at all. The knowledge-based specification thus seems to capture our intuitive requirements for the program more directly and elegantly than the standard specification given.

A standard specification can be viewed as a special case of a knowledgebased specification, one in which the set of systems satisfying it is closed under unions and subsets. It is because of these closure properties that we have the property if a standard program satisfies a standard specification $\sigma$ in a context $\gamma$, then it satisfies it in any restriction of $\gamma$. Clearly, this is not a property that holds of standard programs once we allow knowledgebased specifications. Nevertheless, as the examples above suggest, there is something to be gained - and little to be lost-by allowing the greater generality of knowledge-based specifications. In particular, although we do lose monotonicity, there are other ways of ensuring that safety and liveness properties do hold in the systems of interest.

By forcing us to think in terms of systems, rather than of individual runs, both knowledge-based programs and knowledge-based specifications can be viewed as requiring more "global" thinking than their standard counterparts. The hope is that thinking at this level of abstraction makes the design and specification of programs easier to carry out.

We still need more experience using this framework before we can decide whether this hope will be borne out and whether the knowledge-based approach as described here is really useful. Sanders has other criticisms of the use of knowledge-based programs that I have not addressed here. Very roughly, she provides pragmatic arguments that suggest that we use predicates that have some of the properties of knowledge (for example $K \varphi \Rightarrow \varphi$ ), but not necessarily all of them. This theme is further pursued in [5]. While I 
believe that using predicates that satisfy some of the properties of knowledge will not prove to be as useful as sticking to the original notion of knowledge, we clearly need more examples to better understand the issues.

Besides more examples, as pointed out by Sanders [25], it would also be useful to have techniques for reasoning about knowledge-based programs without having to construct the set of runs generated by the program. In [6], a simple knowledge-based programming language is proposed. Perhaps standard techniques for proving program correctness can be applied to it (or some variant of it). A first step along these lines was taken by Sanders [25], who extended UNITY [2] in such a way as to allow the definition of knowledge predicates (although it appears that the resulting knowledgebased programs are somewhat less general than those described here), and then used proof techniques developed for UNITY to prove the correctness of another knowledge-based protocol for the sequence transmission problem. (We remark that techniques for reasoning about knowledge obtained in CSP programs, but not for knowledge-based programs, were given in 13.) Once we have a number of examples and better techniques in hand, we shall need to carry out a careful evaluation of the knowledge-based approach, and a comparison of it and other approaches. I believe that once the evidence is in, it will show that there are indeed significant advantages that can be gained by thinking at the knowledge level.

Acknowledgments: I would like to thank Ron Fagin, Yoram Moses, Beverly Sanders, and particularly Vassos Hadzilacos, Murray Mazer, Moshe Vardi, and Lenore Zuck for their helpful comments on earlier drafts of the paper. Moshe gets the credit for the observation that knowledge-based protocols do satisfy monotonicity. Finally, I would like to thank Karen Seidel for asking a question at PODC '91 that inspired this paper.

\section{References}

[1] F. Afrati, C. H. Papadimitriou, and G. Papageorgiou. The synthesis of communication protocols. Algorithmica, 3(3):451-472, 1988.

[2] K. M. Chandy and J. Misra. Parallel Program Design: A Foundation. Addison-Wesley, Reading, Mass., 1988.

[3] C. Dwork and Y. Moses. Knowledge and common knowledge in a Byzantine environment: crash failures. Information and Computation, 88(2):156-186, 1990. 
[4] E. A. Emerson and J. Y. Halpern. "Sometimes" and "not never" revisited: on branching versus linear time temporal logic. Journal of the ACM, 33(1):151-178, 1986.

[5] K. Engelhardt, R. van der Meyden, and Y. Moses. Knowledge and the logic of local propositions. In I. Gilboa, editor, Theoretical Aspects of Rationality and Knowledge: Proc. Seventh Conference. Morgan Kaufmann, San Francisco, Calif., 1998.

[6] R. Fagin, J. Y. Halpern, Y. Moses, and M. Y. Vardi. Reasoning about Knowledge. MIT Press, Cambridge, Mass., 1995.

[7] R. Fagin, J. Y. Halpern, Y. Moses, and M. Y. Vardi. Knowledge-based programs. Distributed Computing, 10(4):199-225, 1997.

[8] V. Hadzilacos. A knowledge-theoretic analysis of atomic commitment protocols. In Proc. 6th ACM Symp. on Principles of Database Systems, pages 129-134, 1987.

[9] J. Y. Halpern and R. Fagin. Modelling knowledge and action in distributed systems. Distributed Computing, 3(4):159-179, 1989. A preliminary version appeared in Proc. 4th ACM Symposium on Principles of Distributed Computing, 1985, with the title "A formal model of knowledge, action, and communication in distributed systems: preliminary report".

[10] J. Y. Halpern and Y. Moses. Knowledge and common knowledge in a distributed environment. Journal of the ACM, 37(3):549-587, 1990. A preliminary version appeared in Proc. 3rd ACM Symposium on Principles of Distributed Computing, 1984.

[11] J. Y. Halpern, Y. Moses, and O. Waarts. A characterization of eventual Byzantine agreement. In Proc. 9th ACM Symp. on Principles of Distributed Computing, pages 333-346, 1990.

[12] J. Y. Halpern and L. D. Zuck. A little knowledge goes a long way: knowledge-based derivations and correctness proofs for a family of protocols. Journal of the ACM, 39(3):449-478, 1992.

[13] S. Katz and G. Taubenfeld. What processes know: definitions and proof methods. In Proc. 5th ACM Symp. on Principles of Distributed Computing, pages 249-262, 1986. 
[14] R. Koo and S. Toueg. Effects of message loss on the termination of distributed programs. Information Processing Letters, 27:181-188, 1988.

[15] R. Kurki-Suonio. Towards programming with knowledge expressions. In Proc. 13th ACM Symp. on Principles of Programming Languages, pages 140-149, 1986.

[16] L. Lamport. "Sometimes" is sometimes "not never": on the temporal logic of programs. In Proc. 7th ACM Symp. on Principles of Programming Languages, pages 164-185, 1980.

[17] M. S. Mazer. A link between knowledge and communication in faulty distributed systems. In R. Parikh, editor, Theoretical Aspects of Reasoning about Knowledge: Proc. Third Conference, pages 289-304. Morgan Kaufmann, San Francisco, Calif., 1990.

[18] M. S. Mazer. Implementing distributed knowledge-based protocols. Submitted for publication, 1991.

[19] M. S. Mazer and F. H. Lochovsky. Analyzing distributed commitment by reasoning about knowledge. Technical Report CRL 90/10, DECCRL, 1990.

[20] Y. Moses, D. Dolev, and J. Y. Halpern. Cheating husbands and other stories: a case study of knowledge, action, and communication. Distributed Computing, 1(3):167-176, 1986.

[21] Y. Moses and M. R. Tuttle. Programming simultaneous actions using common knowledge. Algorithmica, 3:121-169, 1988.

[22] G. Neiger and S. Toueg. Simulating real-time clocks and common knowledge in distributed systems. Journal of the ACM, 40(2):334-367, 1993.

[23] A. Neyman. The postive value of information. Games and Economic Behavior, pages 350-355, 1991.

[24] S. Owicki and L. Lamport. Proving liveness properties of concurrent programs. ACM Trans. on Programming Languages and Systems, 4(3):455-495, 1982.

[25] B. Sanders. A predicate transformer approach to knowledge and knowledge-based protocols. In Proc. 10th ACM Symp. on Principles 
of Distributed Computing, pages 217-230, 1991. A revised report appears as ETH Informatik Technical Report 181, 1992. 